\title{
Terminal Disease
}

National Cancer Institute

\section{Source}

National Cancer Institute. Terminal Disease. NCI Thesaurus. Code C9228.

An illness that has a prognosis of death. 